\title{
Workaround Motivation Model (WAMM): An Adaptation of Theory of Interpersonal Behaviour
}

\author{
Nada Nadhrah and Vaughan Michell \\ Business Informatics, Systems and Accounting (BISA), University of Reading, \\ Whitenights Reading UK, RG6 6UD \\ N.Nadhrah@pgr.reading.ac.uk, v.a.michell@henley.reading.ac.uk
}

\begin{abstract}
The study of workarounds (WA) has increased in importance due to their impact on patient safety and efficiency. However, there are no adequate theories to explain the motivation to create and use a workaround in a healthcare sitting. Although theories of technology acceptance help to understand the reasons to accept or reject technology, they fail to explain drivers for alternatives. Also workarounds involve creators and performers that have different motivations. Models such as Theory of Planned Behaviour (TPB) or Theory of Reasoned Action (TRA) can help to explain the role of workaround users, but lack explanation of workaround creators' dynamics. Our aim is to develop a theoretical foundation to explain workaround motivation behaviour models with norms that relate to sanctions to provide an integrated Workaround Motivation Model; WAMM. The development of WAMM model is explained in this paper based on workaround cases as part of further research to establish the model.
\end{abstract}

Keywords: Workaround, Behaviour, Motivation, Healthcare, Patient safety, Consequences, Norm.

\section{Introduction}

Healthcare professionals are continually exposed to new Information Systems (IS) that affects their daily working activities. Many healthcare workers fully use these systems; however, many choose to perform an alternative process or activity manually or via an alternative system. Some have retained old processes, and others have added additional or alternative activities to do their work. Deviations from the formal system or process, i.e. alternative activities are typically addressed in the literature as workarounds (WA) [1], [2], or sometimes deviations from dysfunctional systems [3], [4], [5]. We can define WA as "an alternative work process created by individuals or groups to achieve a benefit over the use of the existing processes" [6]. Many WA involve a single individual alternative and continuous process or action with a single actor or driver [7], Our research identified a classification of four other types. A process WA involves more than one actor working together on a sequence of different activities. A compound WA is a variant of the process WA where more than one continuous process is involved with separate performers. A consequential WA 
exists where the results of a WA drive a further and separate WA process, often used to convert the WA outputs back to a form to feed into the formal process e.g. converting manual documents to automated form. These different types of WA have different motivation impacts referred to later. Many workarounds involve a single actor. However as workarounds are in general unique alternatives to a standard formal process or system they usually have a designer or workaround creator that defines the workaround [9]. This creator is also usually the driver of the workaround. The performers in a workaround if separate from the creator can have a different behaviour in the way they are motivated to execute it. This has implications for the motivation to work around for example we may expect workaround creators motivations to execute the workaround to be higher than performers. These motivation implications will be discussed later. This paper seeks to develop a testable model of the factors affecting motivation to WA. Motivation is 'the reason or reasons one has for acting or behaving in a particular way' [8]. A number of authors have investigated the motivation reasons to perform WA. We classify these into action motives (blocks) and goal motives for WAs [9].

Many authors see positive motivations to WA as a result of a block in normal expected work activities [1]. A block is a system disruption preventing the worker from completing a task or action as desired [1]. For example in order to save time, clinicians try to avoid blocks in the system and have an action motive to work around the block. Block motivated WAs range from the complex design of processes and systems, poor system usability, inadequate user training, inflexible clinical guidelines [1], [10] and slow and time consuming processes [11]. Some users are motivated to create a WA to avoid safety features such as systems produced alarms. These are seen as inconvenient and time consuming. Overriding a system alarm that was designed intentionally to improve patient safety might be considered as a negative WA [10], or deviation and should be eliminated. However, many WAs have a positive safety benefit, For example WAs may be motivated by a physician's superior local and timely event knowledge that improves the safety of the patient [12]. A second motivating group is where there is a personal or professional benefit goal or improvement that could better be achieved by abandoning the formal process or system for a WA [9]. Pernejad cites the example of a new system reducing communication between doctors and nurses ruining their original informal communication and forcing them to WA the system to restore the communications [13]. Overworked clinicians may simply wish to reduce their workload and stress and hence choose to miss out actions or perform alternative actions to those prescribed formally [7], [6]. This often involves reversion to simpler previous habits or norms of behaviour established socially or in a team.

\section{Motivation Models}

Our aim is to identify which factors motivate people to the WA i.e. a general WA model i.e. most relevant factors that drive WA. There are two types - technology acceptance model and general behavioural models. 


\subsection{Technology Acceptance Models}

Technology Acceptance Model (TAM). In TAM Davis [14] suggests the attitude of a potential user of a system has towards using it depends on the how useful they see it (perceived usefulness) and how easy it is to use the system (Perceived ease of use). This results in an attitude and behaviour to use or reject the system. However, we note that a workaround decisions is about accepting or rejecting only a very small part of a system/process and choosing an alternative ad-hoc system/process.

We have seen earlier that WA's are driven by blocks to work or goal motives resulting in improvements/changes to the activity. If an automated system provides a block, effort needs to be increased to use it. We would expect ease of use to be considered low e.g. in the case of lack or training, system complexity, slow systems etc. [9] compared with an alternative system. A system is ideally designed for ease of use e.g. by providing a record log, automatic communication and dissemination of data etc. However, if the automated system is perceived to be less useful personally to an individual they may favour an alternative WA.

TAM tells us why we might not use the automated system, but it does not tell us why we might choose to use a specific WA, manual or automated. TAM is a technology acceptance model and many WA's include both manual and other technology alternatives [7]. We also note that the professional and personal motives for perusing a WA [9] are not covered by TAM. A much deeper understanding of the behaviour is required.

Unified Theory of Acceptance and Use of Technology (UTAUT). The Unified Theory of Acceptance and Use of Technology (UTAUT) proposed by [15] extended TAM to include four new constructs. (1) Performance Expectancy is a broader version of usefulness. (2) Effort Expectancy, a broader version of ease of use identifies the extent of effort involved. (3) Social Influence measures of how much a user is influenced to use the system by people who are socially important to them[16]. Social influence relates to subjective norms of behaviour that influence individual decisions making [17]. Our research suggests social and indeed power influence is important to the workaround decisions. In previous work we identified two types of actors in the WA process, the creator and the performer [7]. Often WA are created and performed by the same person, but in process and compound WA's [9] a separate and often senior creator such as clinical consultants, senior surgeons etc. is present. Creators often allocate the WA to more junior actors to perform. The performer is the individual executes the WA process in order to achieve the task goal. They often have no decision in creating the WA and no choice to participate in it. Hence the social norms for a senior surgeon WA creator are likely to be very different from the junior doctor and different again for the nurse or porter that executes WA and need to be included in the model. (4) Facilitating conditions relates directly to whether the user behaviour is realistic and feasible [18], [19], [15], [20]. For example resources, user, experience knowledge and training. Facilitating conditions relate to many of the drivers of workarounds e.g. the availability of resources, which is known to cause technology blocks [7] or lack of training and experience [7], [21]. 
UTAUT assumes a user can accept or reject technology on a voluntary basis. Very often system use is mandatory and hence some WA's allow for a manual output to be converted back to system inputs, but the motivation for the WA remains. Some senior staff may mandate the WA must be done by virtue of their influence. Social influences, i.e. subjective norms for WA need to include the norms and norm relationships between WA creator and driver and those of the performer. Whilst TAM and UTAUT have limitations in their focus on technology they provide a useful basis for WA behaviour to build on. We now turn to behavioural models to help build the model.

\subsection{Behavioural Models}

We discuss 3 key motivational behaviour models. WA's as deviations are a decisions between the formal obligation to execute a formal process and the option to execute an informal process. We need to understand the use of a system and the extent of freedom to do something different in studying WA. Behavioural models are needed in this context to capture the formal and informal social rules for behaviour and sanctions that influence the selection of WA over the formal process and their consequences.

The Theory of Reasoned Action (TRA) and Theory of Planned Behaviour (TPB). The theory of Planned Behaviour (TPB) is driven from TRA and assumes three determinants to behaviour: : attitude, subjective norm, and perceived behavioural controls [20]. Theory of Planned Behaviour (TPB) is driven from TRA assumes independent determinants of; intention. Attitude and subjective norm, similar to TRA, TAM, UTAUT, but perceived behavioural control (PBC) is an added [22]. Perceived behavioural control relates to the level of influence a user has on the behaviour and their ability to change it- in our case the workaround.

Theory of Interpersonal Behaviour (TIB). Triandis' model considers direct variables useful for workaround behaviour: Intention (I), Habit $(\mathrm{H})$, and Facilitating Conditions (FC). Intention and facilitating conditions were discussed earlier. Habit $(\mathrm{H})$ is "situation-behaviour sequences that are or have become automatic, so that they occur without self-instruction" [19]. Habit is an important factor in WA motivation as many users prefer to use what is familiar to them often in place of the formal system. WA agents often revert back to old habits e.g. manual paper and pen vs automated systems as it is perceived to be easier and less effort [23]. Alternatively they may use newer habits e.g. their iPad in preference to a desktop application [24] or their iPhone as a simpler communication tool in a WA [6].

Indirect variables (via intention) are: Affect (AF), Perceived Consequences (PC), and Social Factors (SF) [19]. Affect, relates to the individual's emotional feelings of pleasure, displeasure, toward a given behaviour [25]. Perceived behavioural consequences relate to the perception of the overall impact/benefit of the action behaviour which is the second determinant of intention [18]. The perception of workaround consequences again relates to the type of actor (creator, performer) discussed earlier. For example, a physician might avoid using a cumbersome system he dislikes by writing a discharge summary manually as a WA and perceive 
consequential benefits of time and effort saved [7]. However, it may be a less happy and enjoyable task for the WA performer who is forced to comply with the WA and convert the text to electronic form. Perceived social norms unlike subjective norms in (TPB) or social factors adopted in UTAUT discussed. It includes two normative dimensions; normative and role beliefs [18]. Normative beliefs consist of the internalisation by an individual of referent people or groups' opinion about executing the behaviour [18], whereas role beliefs refer to the rules of the role or behaviour formally expected of the individual [18]. An additional perceived social norm added to a TIB application is personal normative $(\mathrm{PN})$ belief [26] representing the individual's personal rules of behaviour regarding whether they should perform the behaviour, i.e. do my personal rules suggest I should perform the workaround [18]. Based on the above we use TIB as the base model for WA motivation. Our previous work has identified that WA's are often discouraged and sanctions can exist. Therefore our model needs to include the impact of sanctions on motivation to the workaround. Also our research has identified the importance of the role of professional rules or norms. For example a workaround was justified if it met the professional clinical norms better than the original system [9]. The next section discusses these 2 additional factors.

\subsection{Extension of Consequences and Norms Workaround Factors}

Perceived Behavioural Consequences. Triandis' factor Perceived consequences (PC) refers to the probability that a given consequence or expected benefit will follow from performing the behaviour. The value of the consequence is the "impact attached to the consequence" [19]. The higher the expected value of the act, the more likely the person will intend to perform it [19]. This is an important factor for WA's as a WA is perceived as a 'deviation in medicine with potential risk and safety consequences. Hence the risk must be balanced by a superior benefit. Triandis' model does not differentiate of specify specific consideration and focuses on consequences related to the individual. For example analysis of Gagnon's paper Gagnon, et al. [18] using TIB suggests the questions related to con sequences refer to increased or reduced process time, knowledge and workload and impact on roles.

There are two separate dimensions of consequence/value related to the person, as an individual impact, and related to the process and professional medical benefit [9]. As a workaround is seen as a deviation it can have very high consequences for the individual that need to be considered separately from the benefits regarding process goal. Creating a WA may primarily save physician's time (personal goal) but it might also breach patient confidentiality policy (organisational goal) [6]. This implies a process impact in terms of patient safety and error. A clinician's professional responsibilities ensure the consequential value is not limited to individual's feelings such as satisfied, distressed, and unhappy, but related to a process/professional dimension e.g. may save time and may have a negative e.g. safety impact on the patient. The need to focus on the patient and professional/organisational goals, has been highlighted in the Stafford enquiry in the UK [26]. Unlike most behaviour models that focus on industry the health service is very person focused and safety is critical so we need to include this component. 
The overall value or benefit to the clinician WA user will be determined by their feelings about both personal and professional/organisational value and benefit resulting from the WA. We divide consequences construct into two types: personal value and organisational goal or process impact value. For personal value we measure the individual's expected value in creating/ performing WA e.g. 'for me, creating/ performing WA in my practice would give me the greatest personal benefit in terms of personal WA benefits e.g. save me time, reduce stress, increase my knowledge, increased control be relaxing or satisfying and dissatisfying. So that will cover the positive and negative dimension of WA perceived personal value. The process impact value can be addressed from two benefit perspectives the impact on the process and impact on the individual client or patient. For example, a typical question to test this might be "creating/ performing WA in my practice would provide an overall benefit to professional/organisational goals of'(1) reduce time; (2) allow to update my knowledge; for individual perspective; (3) reduce patients risk; and (4) reduce patient waiting times; (5) improve team work.

Professional Norm (PN). Our research has highlighted that an important motivator of a WA is the perceived professional rules and cultural training which influence clinicians to choose a workaround as it better meets these professional norms than the formal system. For example, there is an error in alternative drug system in pharmacy department: alternative drug is usually drop down list appears to the user when there is certain medicine not available in the stock. Although it has been updated and the alternative medicine is available, yet there is a system error message papers to physicians preventing them from completing the request. They have to call the pharmacist and indicate the problem, so the pharmacist due to their professional norm they will perform the workaround and repeat the request to order alternative drug.

Professional norms are rule based 'components of the professional behaviour 'socialized through education and training, maintained by professional colleges, and have a profound impact on professional work' [27]. We propose two aspects to test this relationship. (a) Questions relating to improvements or reduction in professional standards related to the WA users profession. (b) Questions relating to client or patient benefits.

\section{WAMM an Integrated Framework of Workaround Motivation}

A model of WA motivation must include the key elements of technical (section 2.1above) and behavioural (section 2.2 above) models in addition to perceived consequences and norm modification constructs. This section details the reasoning in developing the Workaround Motivational Model (WAMM). Each of the factors selected will need to be tested in relation to the intention to create or use a WA. As mentioned earlier unlike normal motivation models the WA stakeholder role e.g. as a creator of WA, user and level of use in WA must be recorded as this is expected to reflect the answers to many of the factor questions. 
As mentioned, the theoretical foundation in the model is TIB which involves variables: intention, affect, social factors (normative beliefs and personal beliefs), perceived behavioural consequences, habits, and facilitating conditions. The following hypotheses will need to be tested (1) Affect is a predictor of healthcare professionals' intention to create or perform WA; (2) Perceived consequences are predictors of healthcare professionals' intention to create or perform WA; (3) Perceived social norms are predictors of healthcare professionals' intention to create or perform WA; (4) Personal normative (PN) belief is predictors of healthcare professionals' intention to create or perform WA; (5) Facilitating conditions are predictors of healthcare professionals' intention to create or perform WA; and (6) Habit are predictors of healthcare professionals' intention to create or perform WA (Fig.1).

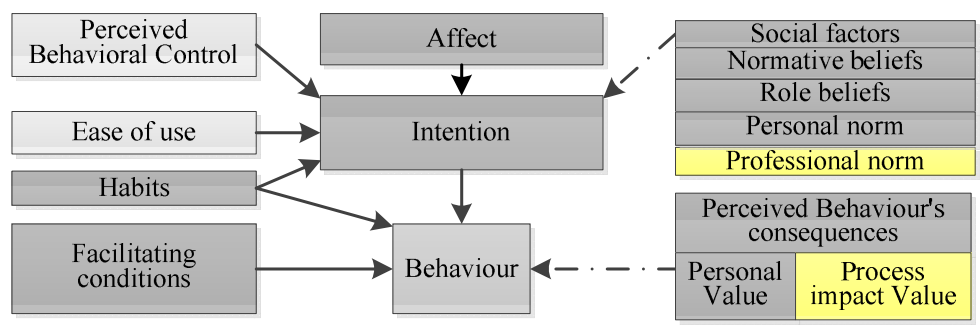

Fig. 1. Workaround Motivational Model (WAMM)

There are four constructs added: perceived behavioural controls (discussed in TPB in section 2.2). Perceived ease of use (discussed in TAM section 2.1), perceived behavioural process value consequences (discussed in section 2.2), and professional norm (discussed in section 2.3). The following hypotheses will be tested as part of the integrated model: (7) perceived behavioural controls is a predictor of healthcare professionals' intention to create or perform WA; (8) Perceived ease of use is a predictor of healthcare professionals' intention to create or perform WA; (9) perceived behavioural process value consequences is a predictor of healthcare professionals' intention to create or perform WA; and (10) Perceived professional norm is a predictor of healthcare professionals' intention to create or perform WA. Hypothesis 7 and 8 are driven from TPB and TAM respectively. Hypothesis 9 and 10 are going to be developed because they are new to the model and needed to be tested.

\section{Case Examples from Practice}

At this point the model questionnaires have been developed and we are in the process of gathering data from questionnaires for analysis of the factors and relationships. Timescales preclude reporting at this stage and hence we demonstrate the use of the model by showing how the variables relate to our previous case studies. The use of the WAMM model can be illustrated by a case example from our research. Many WA's are created by the same individual that performs WA and hence use of WAMM would be expected to be straightforward. However, with more complex WA's such as process and compound WA's there is a creator and separate performers. The WA motivating factors with respect to a creator of a WA are expected to be different to the actors recruited in 
by WA creator to perform the process. We would expect and need to test the possible positive correlations in this activity. For this reason we choose a compound WA case to show how we might expect different results for the WAMM model.

The Discharge summary (DS) report process is a compound WA case study that has been identified in one of the hospitals [6]. The DS is an electronic report that must be written and authenticated within 24 hours from the decision to be discharged by the consultant [6]. Table1 below summarises the activities performed in the DS case vs the positive or negative influence of the motivation factors and how we suggest they may vary in relation to the actor types. There are two types of actors identified: creator and performer. Creator in that case is the physician who initiated and controls the WA process by insisting on writing a discharge report manually rather than via the system. This initiated a chain of activities and involvement by other actors in the process to convert the handwritten report back to the electronic forma in the system to release DS report. From our example case the main motivational factor is likely to be the physician's habit (the case suggested a key motivator for the WA was his habit of writing, not using eth system. The facilitating condition, the factors that make WA easy are likely to be access to and control of the staff needed to convert his manual script back into the format for the system, otherwise this negates the WA benefit. The perceived behavioural controls would be expected to be high motivating factor for the physician as he is responsible and controls the patient's discharge. In terms of affect, we would expect the physician to feel very positive that he does not need to type into the system, but can simply scribble manually at high speed. We would also expect a positive correlation for the personal value impact as the physician has reduced his personal stress in using the system. These points were mentioned by different physicians in reasoning their decision to write in paper sheet rather than the system. We would expect lower or negative correlation in terms of other factors. For example professional norms generally would insist on data entry. Process impact value is likely to be negative as more resources in terms of a nurse and porter are required for the WA and reduce the process benefits.

Table 1. DS workaround case study WAMM measure of expected positive/negative influence

\begin{tabular}{|c|c|c|c|c|c|c|c|c|c|c|}
\hline \multirow[b]{2}{*}{ Actor } & \multirow[b]{2}{*}{ Type } & \multirow[b]{2}{*}{ Activity } & \multirow{2}{*}{$\ll \frac{m}{2}$} & \multirow[b]{2}{*}{ 工 } & \multirow[b]{2}{*}{$\bigcup_{I}$} & \multicolumn{3}{|c|}{ Social norms } & \multirow{2}{*}{$\underset{\frac{1}{2}}{\text { Z }}$} & \multirow[b]{2}{*}{$\vec{a}$} \\
\hline & & & & & & ? & PNB & RNB & & \\
\hline Physician & $\begin{array}{l}\text { Creat } \\
\text { or }\end{array}$ & $\begin{array}{l}\text { Writing DS on a piece of } \\
\text { Paper. }\end{array}$ & ++ & + & + & + & + & + & - & \\
\hline Nurse & $\begin{array}{l}\text { Perfo } \\
\text { rmer }\end{array}$ & $\begin{array}{l}\text { Nurse has to photocopy three } \\
\text { copies. }\end{array}$ & $-\quad-$ & - & - & - & + & + & + & \\
\hline $\begin{array}{l}\text { Medical } \\
\text { secretary }\end{array}$ & $\begin{array}{l}\text { Perfo } \\
\text { rmer }\end{array}$ & $\begin{array}{l}\text { MS has to type the } \\
\text { information from paper into } \\
\text { the computer. }\end{array}$ & $-\quad-$ & - & - & - & + & + & + & $-\quad+$ \\
\hline Porter & $\begin{array}{l}\text { Perfo } \\
\text { rmer }\end{array}$ & $\begin{array}{l}\text { Porter has to transfer one } \\
\text { copy to MS }\end{array}$ & $-\quad-$ & - & - & - & + & + & + & - \\
\hline
\end{tabular}

$\mathrm{AF}=\mathrm{Affect}, \mathrm{PBC}=$ Perceivied Behavioural Controls, $\mathrm{H}=\mathrm{Habit}, \mathrm{FC}=$ Facilitating Conditions, EU= Ease of Use, PNB= Perceived Normative Belifes, RNB= Role Normative Beleifs, PFN=Perceived Professional Norm, PV= Personal Value, PIV= Process Impact Value. 
The performer actors were nurse, medical secretary, and porter. As performers of WA, we would expect different correlations as the users are lower down the power spectrum of the hospital and the nature of some of their work roles e.g. a porter, suggest different motivations from say health professional. We would expect significant motivational variables to be professional norms, and process impact value. Professional norm here was highlighted in a relation to their responsibility to get the job done and the feeling that they must do the WA as it is has become part of their job. The perceived behavioural consequences include personal and process value. We would expect a reasonable positive correlation with process value impact as many of the performers mentioned being motivated to get the job done. Also, perceived social norm were emphasized in the interviews by performers i.e. that 'doctors have asked me to do this WA'. The social norm here is highlighted because doctors must be followed according to the performers in that case. The assumption here is to what extent doctors will be followed and what is the consideration for the WA impact on the patient and the rest of the team working in the hospital whom will affected by those WA behaviours. Space precludes detailing the remaining variables.

\section{Summary and Conclusion}

This paper aimed to develop a theoretical foundation to explain WA motivation among healthcare professional. We explained the structure and different types of WA from the literature and our own research that had a bearing on motivational factors. We reviewed literature related to technology acceptance models and behavioural models used in healthcare context and showed how these models could be adapted to the WA motivation behaviours in healthcare. We identified additional variables to include as: perceived behavioural controls, perceived process value impact as pas part of the perceived consequences construct, and professional norm. We also identified the variation in motivational criteria that depends on the creator vs performer role in WA's. The creator role is an important factor that for obvious reasons is not included in most system and behaviour motivation applications and hence is important to identify. This study explained how these factors were integrated into the Workaround Motivational Model (WAMM). We applied WAMM factors to a compound WA example from our research to explain the type of results we might expect. These will be determined from the results of the questionnaires for creators and performers of workaround types that are currently being gathered ready for analysis. Future work is focused on completing and distributing the questionnaires, in testing heir relation to WA behaviour in healthcare context. We are particularly interested in the differences for compound WA processes and simple single actor workarounds.

\section{References}

1. Halbesleben, J.R., Wakefield, D.S., Wakefield, B.: Work-arounds in health care settings: literature review and research agenda. Health Care Management Review 33(1), 2-12 (2008)

2. Kobayashi, M., Fussell, S.R., Xiao, Y., Seagull, F.: Work coordination, workflow, and workarounds in a medical context. In: CHI 2005 Extended Abstracts on Human Factors in Computing Systems, pp. 1561-1564. ACM (2005) 
3. Morath, J.M., Turnbull, J.: To do no harm. Jossey-Bass, San Francisco (2005)

4. Zhou, X., Ackerman, M., Zheng, K.: CPOE workarounds, boundary objects, and assemblages. In: Proceedings of the 2011 Annual Conference on Human Factors in Computing Systems, pp. 3353-3362. ACM (2011)

5. Reason, J.: Safety in the operating theatre - Part 2: Human error and organisational failure. Quality and Safety in Health Care 14(1), 56-60 (2005)

6. Nadhrah, N., Michell, V.: Measuring physicians WA-Implications on Work Processes and Patient Safety. In: 2013 International Conference on Logistics. Informatics and Services Sciences University of Reading (2013)

7. Nadhrah, N., Michell, V.: A Normative Method to Analyse Workarounds in a Healthcare Environment: their Motivations, Consequences, and Constraints. In: The 14th International Conference on Informatics and Semiotics in Organisations, ICISO 2013, pp. 195-204 (2013)

8. Weiner, B.: Human motivation: Metaphors, theories, and research. SAGE Publications, Incorporated (1992)

9. Nadhrah, N., Michell, V.: Workarounds: Risk or Benevolence for Patient Safety? IGI Global (2014)

10. Vogelsmeier, A.A., Halbesleben, J.R.B., Scott-Cawiezell, J.: Technology implementation and workarounds in the nursing home. Journal of the American Medical Informatics Association 15(1), 114-119 (2008)

11. Ash, J.S., Bates, D.W.: Factors and forces affecting EHR system adoption: report of a 2004 ACMI discussion. Journal of the American Medical Informatics Association 12(1), 8-12 (2005)

12. Niazkhani, Z., Pirnejad, H., van der Sijs, H., Aarts, J.: Evaluating the medication process in the context of CPOE use: the significance of working around the system. International Journal of Medical Informatics 80(7), 490-506 (2011)

13. Pirnejad, H., Niazkhani, Z., van der Sijs, H., Berg, M., Bal, R.: Impact of a computerized physician order entry system on nurse-physician collaboration in the medication process. International Journal of Medical Informatics 77(11), 735-744 (2008)

14. Davis, F.: Perceived usefulness, perceived ease of use, and user acceptance of information technology. MIS quarterly, 319-340 (1989)

15. Venkatesh, V., Morris, M.G., Davis, G.B., Davis, F.: User acceptance of information technology: Toward a unified view. MIS quarterly, 425-478 (2003)

16. Fishbein, M., Ajzen, I.: Belief, attitude, intention and behaviour: An introduction to theory and research. Addison-Wesley (1975)

17. Ajzen, I.: From intentions to actions: A theory of planned behavior. Springer (1985)

18. Gagnon, M.-P., Godin, G., Gagné, C., et al.: An adaptation of the theory of interpersonal behaviour to the study of telemedicine adoption by physicians. International Journal of Medical Informatics 71(2), 103-115 (2003)

19. Bergeron, F., Raymond, L., Rivard, S., Gara, M.-F.: Determinants of EIS use: Testing a behavioral model. Decision Support Systems 14(2), 131-146 (1995)

20. Ajzen, I.: The theory of planned behavior. Organizational behavior and human decision processes 50(2), 179-211 (1991)

21. Ferneley, E.H., Sobreperez, P.: Resist, comply or workaround? An examination of different facets of user engagement with information systems. European Journal of Information Systems 15(4), 345-356 (2006)

22. Fishbein, M., Ajzen, I.: Belief, attitude, intention and behavior: An introduction to theory and research (1975) 
23. Varpio, L., Schryer, C.F., Lehoux, P., Lingard, L.: Working off the record: Physicians' and nurses' transformations of electronic patient record-based patient information. Academic Medicine 81(10), S35-S39 (2006)

24. Workflow concerns and workarounds of readers in an urban safety net teleretinal screening study. In: AMIA Annual Symposium Proceedings. American Medical Informatics Association (2011)

25. Values, attitudes, and interpersonal behavior. In: Nebraska Symposium on Motivation. University of Nebraska Press (1979)

26. Francis, R.: Report of the Mid Staffordshire NHS Foundation Trust Public Inquiry: Executive Summary: TSO Shop (2013)

27. Kirchhoff, J.W.: Norm Systems in Professional Work 